\title{
Intertwining relations of non-stationary Schrödinger operators
}

\author{
F. Cannata*\$ M. Ioffe ${ }^{\dagger}$, G. Junker ${ }^{\ddagger} \|$ and D. Nishnianidze $e^{\dagger * *}$ \\ * Dipartimento di Fisica and INFN, Via Irnerio 46, 40126 Bologna, Italy \\ $\dagger$ Department of Theoretical Physics, University of St. Petersburg, \\ 198904 St. Petersburg, Russia \\ $\ddagger$ Institut für Theoretische Physik, Universität Erlangen-Nürnberg, \\ Staudtstr. 7, D-91058 Erlangen, Germany
}

\begin{abstract}
General first- and higher-order intertwining relations between non-stationary one-dimensional Schrödinger operators are introduced. For the first-order case it is shown that the intertwining relations imply some hidden symmetry which in turn results in a $R$-separation of variables. The Fokker-Planck and diffusion equation are briefly considered. Second-order intertwining operators are also discussed within a general approach. However, due to its complicated structure only particular solutions are given in some detail.
\end{abstract}

\section{Introduction}

It is a basic fact that many physical phenomena are mathematically described by solutions of linear ordinary and partial differential equations. For example, the dynamics of a classical system may be characterized by Newton's equation or equivalently by the EulerLagrange equation. Another example is the dynamics of a non-relativistic quantum system which is governed by a linear differential equation, the well-known Schrödinger equation. Hence, exact solutions of such kind of linear differential equations are of basic interest and, therefore, much effort has been made during the last centuries to find solutions of problems being of the form

$$
\mathcal{L} \psi=0,
$$

where $\mathcal{L}$ denotes some linear differential operator. Here $\psi$ is the wanted solution of (11) in some function space associated with given initial and/or boundary conditions. Many

\footnotetext{
$\S$ E-mail: cannata@bo.infn.it

『E-mail: ioffe@snoopy.phys.spbu.ru

"E-mail: junker@theorie1.physik.uni-erlangen.de

**E-mail: david@heps.phys.spbu.ru
} 
methods have been developed to find such solutions. This paper is concerned with one of them based on an assumed intertwining relation

$$
\mathcal{L}_{1} \mathcal{I}=\mathcal{I} \mathcal{L}_{2}
$$

between two physically relevant differential operators $\mathcal{L}_{1}, \mathcal{L}_{2}$ and the so-called intertwining operator $\mathcal{I}$, which is also assumed to act linearly. This intertwining relation allows to construct a solution for, say, $\mathcal{L}_{1}$ if a solution of $\mathcal{L}_{2}$ is known. To be more precise, let us assume that $\psi_{2}$ is a solution of $\mathcal{L}_{2} \psi_{2}=0$, then due to the intertwining relation $\psi_{1}=\mathcal{I} \psi_{2}$ is a solution of $\mathcal{L}_{1} \psi_{1}=0$. Note that in addition we have to assume that $\psi_{2}$ does not belong to the kernel of $\mathcal{I}$, i.e. $\mathcal{I} \psi_{2} \not \equiv 0$. Multiplying (2) from left and right with the inverse operator $\mathcal{I}^{-1}$ we have $\mathcal{I}^{-1} \mathcal{L}_{1}=\mathcal{L}_{2} \mathcal{I}^{-1}$ and, therefore, one can also start with a given solution $\psi_{1}$ and obtain a new solution $\psi_{2}=\mathcal{I}^{-1} \psi_{1} \not \equiv 0$. However, in many cases the intertwining operator is a differential operator. Therefore, its inverse is a rather complicated integral operator and of less use. This situation, however, changes if in addition we assume that both operators $\mathcal{L}_{1,2}$ are self-adjoint on some common function space, $\mathcal{L}_{1,2}^{\dagger}=\mathcal{L}_{1,2}$. As a consequence one has the adjoint intertwining relation

$$
\mathcal{I}^{\dagger} \mathcal{L}_{1}=\mathcal{L}_{2} \mathcal{I}^{\dagger}
$$

which in addition provides us with the symmetry operators $\mathcal{I I}^{\dagger}$ and $\mathcal{I}^{\dagger} \mathcal{I}$ obeying $\| 1$

$$
\left[\mathcal{L}_{1}, \mathcal{I I}^{\dagger}\right]=0, \quad\left[\mathcal{L}_{2}, \mathcal{I}^{\dagger} \mathcal{I}\right]=0
$$

The above connection between solutions of two differential equations have, to our knowledge, been applied first by Darboux [2] to Sturm-Liouville problems. That is, $\mathcal{L}_{1,2}$ belong to a class of second-order differential operators and may, for example, represent the Hamiltonian of a quantum mechanical degree of freedom in one dimension. This fact may be the reason why most applications of such intertwining relations (sometimes also called Darboux transformations) have been made for eigenvalue problems of stationary Schrödinger Hamiltonians in one dimension. Here, $\mathcal{L}_{1,2}$ represent a pair of Schrödinger Hamiltonians intertwined by a first-order time-independent differential operator. This pair of Hamiltonians together with the intertwining operator (also called supercharge) form the basis of Witten's model of supersymmetric (SUSY) quantum mechanics [3]. It is also closely related to the factorization method of Schrödinger [4] and Infeld and Hull [5], and still finds application in the construction of new potentials giving rise to exactly solvable Schrödinger-eigenvalue problems. See, for example, [6, 7]. This approach has also been generalized [8, 9] to higher-dimensional systems where matrix Hamiltonians necessarily participate in the intertwining relations. Very recently, intertwining relations with supercharges of second (and higher) order in derivatives were investigated for onedimensional [10, 11] and two-dimensional systems [1] as well as for matrix Hamiltonians in one dimension [12]. In the one-dimensional case it was shown that irreducible secondorder transformations, these are those which cannot be represented by two consecutive standard (first-order) Darboux transformations, do exist. In two dimensions second-order irreducible transformations allow to avoid intermediate matrix Hamiltonians. Both, for one-dimensional matrix and two-dimensional scalar quantum systems non-trivial symmetry operators (4) were constructed [1, 12] and led to integrability of the corresponding dynamical systems.

In this paper we will consider intertwining relations of the non-stationary Schrödinger operator

$$
S[V]=i \partial_{t}+\partial_{x}^{2}-V(x, t)
$$


Here $\partial_{t}=\partial / \partial t$ and $\partial_{x}=\partial / \partial x$ denote the partial derivatives with respect to time and position. Throughout this paper we will denote these derivatives, if applied to some function $f$, by a dot and prime, respectively. Hence, we use the notation $\dot{f}(x, t)=$ $\left(\partial_{t} f\right)(x, t)$ and $f^{\prime}(x, t)=\left(\partial_{x} f\right)(x, t)$. It is also evident that we are going to use units such that Planck's constant $2 \pi \hbar$ and the mass $m$ are given by $\hbar=2 m=1$.

Replacing the general operator $\mathcal{L}$ by the Schrödinger operator (5) the corresponding equation (11) reads $S[V] \psi(x, t)=0$ and represents the non-stationary Schrödinger equation for a one-dimensional quantum system under the influence of an external time-dependent potential $V: \mathbb{R} \times \mathbb{R} \rightarrow \mathbb{R},(x, t) \mapsto V(x, t)$. A first investigation of this type of intertwining relations is due to Bagrov and Samsonov [13] who considered a time-dependent first-order intertwining operator. See also the review [14] where in addition a reducible second-order intertwining of non-stationary Schrödinger operators is considered.

In the next section we will reconsider the first-order intertwining and show that, due to the $R$-separation of variables, it provides no basic new results besides those already known from the stationary case of time-independent potentials. This observation has also recently been anticipated by Finkel et al. [15]. However, we additionally show that this fact can be related to some underlying symmetry structure. We will also briefly discuss the time-dependent Fokker-Planck equation in this context and find that this case does not lead to a trivial result in the above sense. In Section 3 we will present particular solutions for stationary potentials intertwined by a non-stationary, i.e. timedependent, second-order operator. We show that the reducible cases (as studied in [14]) are only very special cases of this class. In Section 4 we finally discuss the intertwining of non-stationary Schrödinger operators by non-stationary second-order operators. As special cases we consider the intertwining of the Schrödinger operators corresponding to a non-stationary Hamiltonian with that for a stationary one as well as with that of a timedependent harmonic oscillator. In the concluding remarks in Section 5 we discuss some aspects omitted in the main text such as zero modes, domain question and generalizations to complex potentials.

\section{Intertwining by first-order operators}

In this section we will consider the most general first-order intertwining relations for the Schrödinger operator as defined in (5) and the Fokker-Planck operator $F[U]$,

$$
F[U]=-\partial_{t}+\partial_{x}^{2}+\partial_{x} U^{\prime}(x, t)=-\partial_{t}+\partial_{x}^{2}+U^{\prime}(x, t) \partial_{x}+U^{\prime \prime}(x, t) .
$$

In the latter case, the relation (11) becomes the well-known Fokker-Planck equation [16] characterizing the diffusion of a macroscopic (one-dimensional) degree of freedom in an external time-dependent drift potential $U: \mathbb{R} \times \mathbb{R} \rightarrow \mathbb{R},(x, t) \mapsto U(x, t)$ with a diffusion constant set equal to unity. For both cases we start with the most general intertwining operator of first order given by

$$
q^{+}(x, t)=\xi_{0}(x, t) \partial_{t}+\xi_{1}(x, t) \partial_{x}+\xi_{2}(x, t)
$$

with, in general, complex-valued functions $\xi_{0}, \xi_{1}$ and $\xi_{2}$. Note that in contrast to [13] we also allow for a first-order operator in $\partial_{t}$. 


\subsection{The Schrödinger operator and separation of variables}

For the above defined Schrödinger operator (5) the most general first-order intertwining relation reads

$$
S\left[V_{1}\right] q^{+}=q^{+} S\left[V_{2}\right],
$$

where the functions $\xi_{i}(i=0,1,2)$ and the potentials $V_{1,2}$ are clearly not independent of each other. In fact, we will now consider the question: Which $\xi$ 's lead to real-valued potentials and how are the latter connected with the them?

Inserting the explicit forms of the Schrödinger operators (5) and the intertwining operator (7) into relation (8) and equating the coefficients of identical (partial) differential operators we immediately find that $\xi_{0}$ and $\xi_{1}$ may only depend on time, i.e. $\xi_{0}^{\prime}=0=\xi_{1}^{\prime}$. The assumption that $\xi_{0}$ does not vanish identically then leads to the conclusion that the potential difference $V_{1}-V_{2}$ does depend only on time. This is a rather trivial casef and, therefore, we will set $\xi_{0} \equiv 0$ without loss of generality.[2] Making now the following reparametrizations $\xi_{1}(t)=e^{i \beta(t)} \rho(t)$ and $\xi_{2}(x, t)=e^{i \beta(t)} \rho(t) \chi^{\prime}(x, t)$ with $\beta: \mathbb{R} \rightarrow \mathbb{R}$, $\rho: \mathbb{R} \rightarrow \mathbb{R}^{+}$and $\chi: \mathbb{R} \times \mathbb{R} \rightarrow \mathbb{C}$ we find

$$
\begin{aligned}
& V_{1}(x, t)=\chi^{\prime 2}(x, t)+\chi^{\prime \prime}(x, t)-i \dot{\chi}(x, t)+\alpha(t)-\dot{\beta}(t)+i \dot{\rho}(t) / \rho(t) \\
& V_{2}(x, t)=\chi^{\prime 2}(x, t)-\chi^{\prime \prime}(x, t)-i \dot{\chi}(x, t)+\alpha(t)
\end{aligned}
$$

where $\alpha$ is some time-dependent complex-valued integration constant. Following the argumentation made in footnote 1 we may set $\beta \equiv 0$ without loss of generality. Furthermore, we may also set $\alpha \equiv 0$ because it can always be absorbed in $\chi$ via the substitution $\chi \rightarrow \chi-i \int d t \alpha$. Hence, we are left with

$$
\begin{aligned}
& V_{1}(x, t)=\chi^{\prime 2}(x, t)+\chi^{\prime \prime}(x, t)-i \dot{\chi}(x, t)+i \dot{\rho}(t) / \rho(t), \\
& V_{2}(x, t)=\chi^{\prime 2}(x, t)-\chi^{\prime \prime}(x, t)-i \dot{\chi}(x, t) .
\end{aligned}
$$

Here the so-called superpotential $\chi$ is still not arbitrary as the potentials are assumed to be real-valued. This can, for example, be achieved by assuming a stationary real-valued superpotential. This, however, leads to the standard stationary supersymmetric quantum mechanics discussed to a large extend during the last two decades [3]. Therefore, we will consider a complex-valued superpotential

$$
\chi(x, t)=h(x, t)+i g(x, t)
$$

with real-valued functions $h$ and $g \not \equiv 0$. The reality condition $\operatorname{Im} V_{1}=\operatorname{Im} V_{2}=0$ then leads to

$$
2 g^{\prime \prime}+\dot{\rho} / \rho=0, \quad 2 h^{\prime} g^{\prime}-g^{\prime \prime}-\dot{h}=0,
$$

which can easily be integrated to

$$
\begin{aligned}
& g(x, t)=-\frac{1}{4} \frac{\dot{\rho}(t)}{\rho(t)} x^{2}+\frac{1}{2} \rho(t) \dot{\mu}(t) x+\gamma(t), \\
& h(x, t)=\frac{1}{2} \ln \rho(t)+K(x / \rho(t)+\mu(t)),
\end{aligned}
$$

\footnotetext{
${ }^{1}$ Note that from $V_{2}(x, t)-V_{1}(x, t)=f(t)$ and $S\left[V_{1}\right] \psi_{1}=0$ follows that $S\left[V_{2}\right] \psi_{2} \equiv S\left[V_{1}+f\right] \psi_{2}=0$ has the solution $\psi_{2}(x, t)=\exp \left\{i \int_{0}^{t} d \tau f(\tau)\right\} \psi_{1}(x, t)$. That is, knowing a normalized solution of the time-dependent Schrödinger equation for $V_{1}$ one immediately has a solution for $V_{2}$, too, which is also normalized. Complex-valued $f$ 's are not allowed as both, $V_{1}$ and $V_{2}$, are assumed to be real-valued.

${ }^{2} \mathrm{~A}$ posteriori, this justifies the ansatz made in 13 .
} 
where $\mu$ and $\gamma$ are arbitrary real-valued function of time and $K$ is an arbitrary real-valued function of the variable $y=x / \rho+\mu$. In terms of these functions the final form of the two partner potentials is

$V_{1,2}(x, t)=\frac{1}{\rho^{2}(t)}\left[K^{\prime 2}(y) \pm K^{\prime \prime}(y)\right]-\frac{\ddot{\rho}(t)}{4 \rho(t)} x^{2}+\left(\dot{\rho}(t) \dot{\mu}(t)+\frac{\rho(t) \ddot{\mu}(t)}{2}\right) x-\frac{\rho^{2}(t) \dot{\mu}^{2}(t)}{4}+\dot{\gamma}(t)$

and the intertwining operator reads

$$
q^{+}(x, t)=\rho(t) \partial_{x}+K^{\prime}(x / \rho(t)+\mu(t))-\frac{i}{2}\left(\dot{\rho}(t) x-\rho^{2}(t) \dot{\mu}(t)\right) .
$$

It is obvious that with appropriate choices for $K, \rho, \mu$ and $\gamma$ at least one of the two potentials (14) can be made stationary. We will not investigate this aspect here but rather like to discuss a more general property of these quantum systems, the so-called $R$-separation of variables.

In doing so, let us demonstrate that the non-stationary Schrödinger equation

$$
S\left[V_{1,2}\right] \psi_{1,2}(x, t) \equiv\left(i \partial_{t}+\partial_{x}^{2}-V_{1,2}\right) \psi_{1,2}(x, t)=0
$$

with potentials as given in (14), which is equivalent to the intertwining (8), admits a separation of variables. In fact, under the transformation

$$
y=x / \rho(t)+\mu(t), \quad \phi_{1,2}(y, t)=\sqrt{\rho(t)} e^{i g(x, t)} \psi_{1,2}(x, t)
$$

this Schrödinger equation becomes [17]

$$
i \rho^{2}(t) \partial_{t} \phi_{1,2}(y, t)=\left[-\partial_{y}^{2}+K^{\prime 2}(y) \pm K^{\prime \prime}(y)\right] \phi_{1,2}(y, t),
$$

which is obviously separable in $y$ and $t$. Hence, the solutions of the original problem (16) have the general form $\psi(x, t)=\tilde{R}(y, t) Y(y) T(t)$ which is known as the $R$-separation of variables [18]. In other words, for any pair of Schrödinger operators $S\left[V_{1,2}\right]$, which admits a first-order intertwining relation (8) there exists a transformation (17) to some new coordinate in which the potentials become stationary and form the well-known pair of stationary SUSY partner potentials [3]. As a consequence nothing essentially new emerges from the first-order intertwining of the non-stationary Schrödinger operator. This fact has also recently been observed by Finkel et al. [15]. Note that the transformation associated with the special case $\rho(t)=1$ and $\mu(t)=v t$ with constant velocity $v$ corresponds to the Galilei transformation. See, for example, the textbook by Galindo and Pascual [19].

Here let us add that this $R$-separation of variables can be related to the existence of symmetry operators for the two Schrödinger operators in questions. First, we note that with the results above one can directly verify the adjoint intertwining relation

$$
q^{-} S\left[V_{1}\right]=S\left[V_{2}\right] q^{-}
$$

where

$$
q^{-} \equiv\left(q^{+}\right)^{\dagger}=-\rho(t) \partial_{x}+K^{\prime}(x / \rho(t)+\mu(t))+\frac{i}{2}\left(\dot{\rho}(t) x-\rho^{2}(t) \dot{\mu}(t)\right) .
$$

In other words, in analogy to the discussion of (3) the Schrödinger operator $S[V]$ and in particular its time-derivative $i \partial_{t}$ can be viewed as self-adjoint operators on a suitable domain, $\left(i \partial_{t}\right)^{\dagger}=i \partial_{t}$. Then from (8) and (19) immediately follows

$$
\left[S\left[V_{1}\right], q^{+} q^{-}\right]=0, \quad\left[S\left[V_{2}\right], q^{-} q^{+}\right]=0,
$$


which is a special case of (4). This discussion suggests that the reverse conclusion may also be true. In fact we are able to prove the following

Theorem: Any Schrödinger operator $S[V]$ (with real-valued potential $V$ ) having a selfadjoint symmetry operator $R$, obeying $[S[V], R]=0$ and being quadratic in $\partial_{x}$, admits the $R$-separation of variables for the corresponding time-dependent Schrödinger equation and an intertwining relation of first order with its superpartner.

For the proof of this theorem we start with the most general quadratic and self-adjoint ansatz for the symmetry operator,

$$
R(x, t)=-\omega(t) \partial_{x}^{2}+i\left\{\delta(x, t), \partial_{x}\right\}+\zeta(x, t),
$$

obeying the symmetry condition

$$
[S[V], R] \equiv\left[i \partial_{t}+\partial_{x}^{2}-V(x, t), R(x, t)\right]=0 .
$$

Here, $\delta: \mathbb{R} \times \mathbb{R} \rightarrow \mathbb{R}$ and $\zeta: \mathbb{R} \times \mathbb{R} \rightarrow \mathbb{R}$ are arbitrary real functions of position and time, whereas $\omega: \mathbb{R} \rightarrow \mathbb{R}^{+}$is, without loss of generality, assumed to be an arbitrary strictly positive function of time only. The coefficient functions of the symmetry operator (22) are clearly related with the potential $V$ due to the symmetry condition (23) giving rise to

$$
\delta(x, t)=\frac{1}{4} \dot{\omega}(t) x+\frac{1}{2} \nu(t), \quad \zeta(x, t)=\Phi(z)+\frac{\dot{\omega}^{2}(t)}{16 \omega(t)} x^{2}+\frac{\nu(t) \dot{\omega}(t)}{4 \omega(t)} x,
$$

where $\Phi$ is an arbitrary real function of the variable

$$
z=\frac{x}{\sqrt{\omega(t)}}-\int_{0}^{t} d \tau \frac{\nu(\tau)}{\omega^{3 / 2}(\tau)}
$$

and $\nu$ is another arbitrary real function of time. In terms of these functions the potential must take the form

$$
V(x, t)=-\frac{1}{8 \omega(t)}\left(\ddot{\omega}(t)-\frac{\dot{\omega}^{2}(t)}{2 \omega(t)}\right) x^{2}-\frac{1}{2 \omega(t)}\left(\dot{\nu}(t)-\frac{\nu(t) \dot{\omega}(t)}{2 \omega(t)}\right) x+\frac{\Phi(z)}{\omega(t)} .
$$

We note that the $R$-separation of variables for the Schrödinger equation $S[V] \psi(x, t)=0$ becomes explicit by making the change of variables

$$
\phi(z, t)=\omega^{1 / 4}(t) \exp \left\{-i\left(\frac{\dot{\omega}(t)}{8 \omega(t)} x^{2}+\frac{\nu(t)}{2 \omega(t)} x-\frac{1}{4} \int_{0}^{t} d \tau \frac{\nu^{2}(\tau)}{\omega^{2}(\tau)}\right)\right\} \psi(x, t),
$$

which results in

$$
\left(i \omega(t) \partial_{t}+\partial_{z}^{2}+\Phi(z)\right) \phi(z, t)=0
$$

Let us remark that the symmetry operator $R$ of the form (22) with coefficient functions related by (24) is factorizable, i.e. $R=\tilde{q}^{+} \tilde{q}^{-} \geq 0$ with $\tilde{q}^{-}=\left(\tilde{q}^{+}\right)^{\dagger}$. The supercharge $\tilde{q}^{+}$ has again the form (15) if the following substitutions are made:

$$
\rho(t) \rightarrow \omega^{1 / 2}(t), \quad \dot{\mu}(t) \rightarrow-\frac{\nu(t)}{\omega^{3 / 2}(t)}, \quad y \rightarrow z, \quad K^{\prime 2}+K^{\prime \prime} \rightarrow \Phi
$$

Thus we conclude that if some non-stationary Schrödinger operator $S[V]$ has a symmetry characterized by a second-order operator of the form (22) it admits the $R$-separation of variables and the superpartner potential of $V$ can also be constructed. The nonstationary Schrödinger operator associated with this superpartner has again a positive symmetry operator, which in this case is given by $\tilde{q}^{-} \tilde{q}^{+}$. 


\subsection{The Fokker-Planck equation}

In this subsection we will now consider the most general first-order intertwining relations allowing to construct from known solutions of one Fokker-Planck equation solutions of its corresponding superpartner. In order to keep as close as possible to the previous discussion we will first transform the Fokker-Planck equation into a diffusion (or imaginary-time Schrödinger) equation. Indeed, it is well-known [16] that the Fokker-Planck equation $F[U] P(x, t)=0$ transforms under the substitution $P(x, t)=\exp \{-U(x, t) / 2\} \psi(x, t)$ into the diffusion equation

$$
D[V] \psi(x, t) \equiv\left(-\partial_{t}+\partial_{x}^{2}-V(x, t)\right) \psi(x, t)=0,
$$

where the potential $V$ is given in terms of the drift potential $U$ in the following way

$$
V(x, t)=\frac{1}{4} U^{\prime 2}(x, t)-\frac{1}{2} U^{\prime \prime}(x, t)-\frac{1}{2} \dot{U}(x, t) .
$$

Note that the diffusion operator $D[V]$ can be obtained from the corresponding Schrödinger operator $S[V]$ via a Wick rotation $i \partial_{t} \rightarrow-\partial_{t}$. There are, however, essential differences from the physical point of view as the solution $P$ of the Fokker-Planck equation should represent a probability distribution. As a consequence $\psi(x, t)=\exp \{U(x, t) / 2\} P(x, t)$ is also required to be real and positive.

We are now interested in finding the most general first-order intertwining operator $q^{+}$ as given in (17) obeying the relation

$$
D\left[V_{1}\right] q^{+}=q^{+} D\left[V_{2}\right] \quad \Longleftrightarrow \quad F\left[U_{1}\right] e^{U_{1} / 2} q^{+} e^{-U_{2} / 2}=e^{U_{1} / 2} q^{+} e^{-U_{2} / 2} F\left[U_{2}\right] .
$$

Here we closely follow the derivations of the previous subsection and the result is identical to that given in (9) after Wick rotation. Again we may set $\alpha \equiv 0$ as it can be absorbed

in $\chi$. Furthermore, as noted above, the intertwining operator $q^{+}=e^{i \beta} \rho\left(\partial_{x}+\chi^{\prime}\right)$ should map a positive solution of $D\left[V_{2}\right] \psi_{2}=0$ into a positive solution of $D\left[V_{1}\right] \psi_{1}=0$, i.e. $\psi_{1}=q^{+} \psi_{2} \geq 0$. This leads us to the conclusion that $\beta$ must vanish identically and $\chi$ has to be real-valued. Hence, we are left with

$$
\begin{aligned}
& V_{1}(x, t)=\chi^{\prime 2}(x, t)+\chi^{\prime \prime}(x, t)+\dot{\chi}(x, t)-\dot{\rho}(t) / \rho(t), \\
& V_{2}(x, t)=\chi^{\prime 2}(x, t)-\chi^{\prime \prime}(x, t)+\dot{\chi}(x, t),
\end{aligned}
$$

giving rise, cf. (31), to the following form of the drift potentials

$$
\begin{aligned}
& U_{1}(x, t)=2 \ln \rho(t)-2 \chi(x, t), \\
& U_{2}(x, t)=2 \chi(x,-t) .
\end{aligned}
$$

In contrast to our result in the previous subsection we find here that $\chi$ has to be a real-valued function which, however, is a function of the two independent variables $x$ and $t$. Therefore, the Fokker-Planck case can, in contrast to the Schrödinger case, not be transformed into a stationary problem. This seems to be related to the fact that the diffusion operator is, in contrast to the Schrödinger operator, not self-adjoint. Consequently, the adjoint intertwining relation (3) and the resulting symmetries (4) do not exist. Physically, this is due to the time-reversal symmetry, which exists in the case of the Schrödinger equation [19] but not for the Fokker-Planck and diffusion equation. This is also explicated in the above result (34) which shows that the two SUSY-partner drift potentials are essentially related via a time reversal. The additional difference in an overall sign between them is already known from the stationary case [3]. 


\section{Second-order intertwining for stationary potentials: particular solutions and symmetry operators}

Recently higher-order generalizations of the SUSY-intertwining relations for stationary Schrödinger operators have been investigated to some extend [10, 11]. In the 1-dimensional case it was shown [11] that in general the intertwining of a pair of stationary Schrödinger operators by a second-order differential operator cannot be reduced to two consecutive transformations with some intermediate self-adjoint Hamiltonian. In the 2-dimensional case second-order intertwining operators also allow to intertwine pairs of standard stationary Schrödinger Hamiltonians with scalar potential [1]. All of these self-adjoint Hamiltonians have a hidden symmetry, cf. (4), characterized by a differential operator.

In the remaining part of this paper we will investigate the intertwining of a pair of Schrödinger operators $S\left[V_{1}\right]$ and $S\left[V_{2}\right]$ by second-order intertwining operators of the form:

$$
q^{+}(x, t)=G(x, t) \partial_{x}^{2}-2 F(x, t) \partial_{x}+B(x, t) .
$$

As in the first-order case it can be shown that the inclusion of an additional term being of first order in $\partial_{t}$ leads to the trivial situations where the difference $V_{1}-V_{2}$ depends on the time $t$ only, cf. footnote 1. Furthermore, from the intertwining relation (8) with above $q^{+}$ one can conclude that the function $G$ may not depend on $x$ and similar to the discussion in the previous section it is even possible to exclude a phase. In other words, without loss of generality we have $G(x, t) \equiv g(t)$, which should not be confused with $g$ used previously, and consider from now on an intertwining operator of the form

$$
q^{+}(x, t)=g(t) \partial_{x}^{2}-2 F(x, t) \partial_{x}+B(x, t) .
$$

We are unable to find the general analytic solutions of the intertwining relation (8) with $q^{+}$as given above. Therefore, in this and the next section we will construct only some particular solutions of interest. More precisely, in this section we shall look for the solutions of the intertwining relation (8) for the case where both potentials $V_{1}$ and $V_{2}$ are stationary, i.e. do not depend on $t$. It is evident that one class of solutions is already known from [11]. Assuming a supercharge $q^{+}$whose coefficient functions are real and do not depend on $t$, it follows that the corresponding solutions of (8) will coincide with those of the stationary intertwining relations $\left(-\partial_{x}^{2}+V_{1}(x)\right) q^{+}(x)=q^{+}(x)\left(-\partial_{x}^{2}+V_{2}(x)\right)$ which can be found in [11]. Here we are interested in more general solutions of (8) with operators $q^{+}$depending manifestly on $t$. That is, we will search for particular solutions of the intertwining relation

$$
\left(i \partial_{t}-H_{1}\right) q^{+}(x, t)=q^{+}(x, t)\left(i \partial_{t}-H_{2}\right)
$$

with standard stationary Hamiltonians $H_{1,2}=-\partial_{x}^{2}+V_{1,2}(x)$ but an explicit time-dependent intertwining operator.

\subsection{Systems with symmetry operators of third order}

A first suitable ansatz with simple $t$-dependence in (36) is

$$
q^{+}(x, t)=M^{+}(x)+A(t) a^{+}(x)
$$


where we have set

$$
M^{+}(x) \equiv \partial_{x}^{2}-2 f(x) \partial_{x}+b(x), \quad a^{+}(x) \equiv \partial_{x}+W(x) .
$$

Here all functions besides $A$ are considered to be real valued $]^{f}$. With this ansatz the intertwining relation (37) results in the condition

$$
i \dot{A} a^{+}=H_{1} M^{+}-M^{+} H_{2}+A\left(H_{1} a^{+}-a^{+} H_{2}\right) .
$$

Equating the coefficient of identical powers in $\partial_{x}$ on both sides of (40) one obtains the following system of equations:

$$
\begin{aligned}
& i \dot{A}=2 \tilde{m}+2 m A, \\
& H_{1} M^{+}-M^{+} H_{2}=2 \tilde{m} a^{+}, \\
& H_{1} a^{+}-a^{+} H_{2}=2 m a^{+},
\end{aligned}
$$

with real constants $\tilde{m}$ and $m$. For our further discussion we will consider the two cases $m \neq 0$ and $m=0$ separately.

a) Solutions for $m \neq 0$ :

In this case the first equation in (41) immediately leads to $A(t)=m_{0} e^{-2 i m t}-\tilde{m} / m$, $m_{0} \in \mathbb{R}$, and results in an intertwining operator of the form

$$
q^{+}(x, t)=\partial_{x}^{2}-\left(2 f(x)+\frac{\tilde{m}}{m}\right) \partial_{x}+b(x)-\frac{\tilde{m}}{m} W(x)+m_{0} e^{-2 i m t} a^{+}(x) .
$$

It is obvious that without loss of generality we may set $\tilde{m}=0$ as a non-vanishing $\tilde{m}$ may always be absorbed via a proper redefinition of $f$ and $b$. As a consequence, the second relation in (41) leads to a second-order intertwining between $H_{1}$ and $H_{2}$. This has already been considered in [11] and it was found that the potentials $V_{1}, V_{2}$ and the function $b$ can be expressed in terms of $f$ and two arbitrary real constants $a$ and $d$ :

$$
\begin{aligned}
& V_{1,2}(x)=\mp 2 f^{\prime}(x)+f^{2}(x)+\frac{f^{\prime \prime}(x)}{2 f(x)}-\frac{f^{\prime 2}(x)}{4 f^{2}(x)}-\frac{d}{4 f^{2}(x)}-a, \\
& b(x)=-f^{\prime}(x)+f^{2}(x)-\frac{f^{\prime \prime}(x)}{2 f(x)}+\frac{f^{\prime 2}(x)}{4 f^{2}(x)}+\frac{d}{4 f^{2}(x)} .
\end{aligned}
$$

Finally we note that the last equation in (41) coincides with the usual first-order intertwining relation of SUSY quantum mechanics between the Hamiltonians $H_{1}$ and $H_{2}+2 m$. Therefore, the potentials can be expressed in terms of the SUSY potential $W$ as follows [3]:

$$
V_{1}(x)=W^{2}(x)+W^{\prime}(x), \quad V_{2}(x)=W^{2}(x)-W^{\prime}(x)-2 m .
$$

Comparing this with (43) we conclude that $W(x)=-2 f(x)-m x$ and $f$ must satisfy the so-called Painleve IV equation:

$$
f^{\prime \prime}=\frac{f^{\prime 2}}{2 f}+6 f^{3}+8 m x f^{2}+2\left(m^{2} x^{2}-m+a\right) f+\frac{d}{2 f} .
$$

As an example we mention here that for $d=-a^{2}$ it can be shown that any $f$ obeying the generalized Riccati equation $f^{\prime}=-2 f^{2}-2 m x f-a$ is also a solution of (45). The

\footnotetext{
${ }^{3}$ We also assume $A \not \equiv 0$, as the case $A \equiv 0$ has already been studied in [11].
} 
general solution of this generalized Riccati equation and the corresponding potentials (44) are discussed in detail in [6], which also shows their connection to the most general SUSY partners of the harmonic-oscillator potential. Note that cases with $d<0$ are reducible ones [11].

We have already discussed in the previous section that the intertwining relation (37) and its adjoint give rise to the symmetry operators $q^{+} q^{-}$and $q^{-} q^{+}$for $\left(i \partial_{t}-H_{1}\right)$ and $\left(i \partial_{t}-H_{2}\right)$, respectively. For the present case of stationary potentials one can use results of [11] to show that these symmetry operators (defined up to a square polynomial in $H_{1,2}$ ) have the explicit form

$$
\begin{aligned}
& R_{1}(x, t)=e^{2 i m t} M^{+}(x) a^{-}(x)+e^{-2 i m t} a^{+}(x) M^{-}(x), \\
& R_{2}(x, t)=e^{2 i m t} a^{-}(x) M^{+}(x)+e^{-2 i m t} M^{+}(x) a^{-}(x),
\end{aligned}
$$

where $a^{-}=\left(a^{+}\right)^{\dagger}$ and $M^{-}=\left(M^{+}\right)^{\dagger}$. Let us note that these results have also been found recently by Fushchych and Nikitin [20] using a different approach.

b) Solutions for $m=0$ :

In this case it follows from (41) that $A(t)=-2 i \tilde{m} t$ where, without loss of generality, we have set the integration constant $A(0)$ to zero. The remaining two conditions of (41) lead to

$$
\begin{aligned}
& V_{1,2}(x)=W^{2}(x) \pm W^{\prime}(x), \quad f(x)=n-\frac{1}{2} W(x), \\
& b(x)=\frac{1}{2}\left(W^{\prime}(x)-W^{2}(x)\right)-2 n W(x)-\tilde{m} x
\end{aligned}
$$

where $W$ is a solution of the Painleve II equation

$$
W^{\prime \prime}=2 W^{3}+4 \tilde{m} x W+k
$$

and $n, k$ are real constants. Here we note that an additional integration constant appearing on the right-hand side of the last relation in (47) has been set to zero as it can always be absorbed via a proper redefinition of the independent variable $x$.

Again let us mention that for $k=-4 \tilde{m}$ a particular solution of this equation reads $W(x)=1 / x$ giving rise to inverse-squared potentials $V_{1,2}$. On the other hand, for $k=2 \tilde{m}$ a solution of the ordinary Riccati equation $W^{\prime}=W^{2}+k x$ is also a solution of (48). Note that such solutions can be expressed in terms of Bessel functions [21].

As in the previous case the intertwining relation (37) leads to symmetry operators, which can also be reduced to third-order differential operators:

$$
\begin{aligned}
& R_{1}=M^{+} M^{-}-H_{1}^{2}+2 i \tilde{m} t\left(M^{+} a^{-}-a^{+} M^{-}\right)+4 \tilde{m}^{2} t^{2} H_{1}, \\
& R_{2}=M^{-} M^{+}-H_{2}^{2}+2 i \tilde{m} t\left(a^{-} M^{+}-M^{-} a^{+}\right)+4 \tilde{m}^{2} t^{2} H_{2} .
\end{aligned}
$$

However, in addition to that, because of $m=0$, we can even construct another pair of third-order operators commuting with $\left(i \partial_{t}-H_{1,2}\right)$. Indeed, the last two equations of (41) can be rearranged as follows

$$
\left[H_{1}, M^{+} a^{-}\right]=2 \tilde{m} H_{1}, \quad\left[H_{2}, a^{-} M^{+}\right]=2 \tilde{m} H_{2}
$$

and directly lead to

$$
\left[i \partial_{t}-H_{1}, i M^{+} a^{-}+2 \tilde{m} t H_{1}\right]=\left[i \partial_{t}-H_{2}, i a^{-} M^{+}+2 \tilde{m} t H_{2}\right]=0,
$$


Hence, besides those given in (49), we have found an additional hermitean pair of symmetry operators:

$$
\begin{aligned}
& \tilde{R}_{1}=i\left(M^{+} a^{-}-a^{+} M^{-}\right)+4 \tilde{m} t H_{1}=\dot{R}_{1} / 2 \tilde{m} \\
& \tilde{R}_{2}=i\left(a^{-} M^{+}-M^{-} a^{+}\right)+4 \tilde{m} t H_{2}=\dot{R}_{2} / 2 \tilde{m} .
\end{aligned}
$$

Whereas these operators have already been given in [20] the previous pair (49) is a new, independent one, which does not commute with (52).

\subsection{Systems with symmetry operators of fourth order}

As a second ansatz for the intertwining relation (37) we consider an operator with two coefficient functions depending manifestly on time:

$$
q^{+}(x, t)=\theta(t) M^{+}(x)+i \lambda(t) x a^{+}(x),
$$

where $\theta$ and $\lambda$ are assumed to be real-valued functions and the operators $M^{+}$and $a^{+}$are of the same form as in (39). For this ansatz the intertwining relation (37) leads to

$$
\begin{aligned}
& \dot{\theta}=-2 \lambda, \quad \dot{\lambda}=\beta \theta, \\
& 2 M^{+}=x a^{+} H_{2}-H_{1} x a^{+}, \quad \beta x a^{+}=M^{+} H_{2}-H_{1} M^{+} .
\end{aligned}
$$

The first two equations, containing the real constant $\beta$, can easily be integrated and yield, for $\beta>0$, oscillatory solutions. Furthermore, with the above conditions the potentials $V_{1,2}$ and the functions $W$ and $b$ can exclusively be expressed in terms of the function $f$ :

$$
\begin{aligned}
W(x)= & -2 f(x)+\frac{c}{x}, \quad b(x)=f^{\prime}(x)+2 f^{2}(x)-V_{2}(x)+(\beta / 4) x^{2}+a_{0}, \\
V_{1,2}(x)= & \mp 2 f^{\prime}(x)+4 f^{2}(x)+\frac{2(1-2 c) f(x)}{x}+\frac{\beta}{8} x^{2}+\frac{4(c-1) f(x)}{x^{2}} \\
& -\frac{2}{x^{2}} \int_{x_{0}}^{x} d z\left[f(z)-2 z f^{2}(z)\right]+a_{0},
\end{aligned}
$$

where $a_{0}, c$ and $x_{0}$ are some real constants and the function $f$ must satisfy the non-linear third-order differential equation:

$$
-2 f V_{2}^{\prime}+4 b f^{\prime}+f^{\prime \prime \prime}+4 f^{\prime 2}+4 f f^{\prime \prime}+2 \beta x f-\beta c+\beta / 2=0
$$

This equation is certainly too complicate to solve for arbitrary values of the constants $\beta$ and $c$. However, for $c=0$ and an arbitrary $d$ one can show that all $f$ 's satisfying the Riccati equation

$$
f^{\prime}(x)=2 f^{2}(x)-(\beta / 4) x^{2}+d
$$

are also solutions of (56). Such solutions correspond to Hamiltonians obeying the intertwining relation $\mathrm{H}_{1} \mathrm{a}^{+}=a^{+} \mathrm{H}_{2}$ in addition to (54).

It is straightforward to show that there are no other values of the constant $c$ for which the Hamiltonians $H_{1,2}$ are intertwined by operators of first or second order. Since solutions of eq. (57) do not exhaust the solutions of (56) we believe that, at least in some cases, operators $q^{+}$of the form (53) intertwine the Schrödinger operators $S\left[V_{1,2}\right]$ but not the Hamiltonians $H_{1,2}$.

\footnotetext{
${ }^{4}$ We exclude here the case $\beta=0$ which corresponds to the intertwining of $H_{1,2}$ by $M^{+}$.
} 


\subsection{Reducible and irreducible second order intertwining.}

The natural question we will now consider concerns the reducibility of the second-order intertwining operator (36) to a pair of consecutive first-order operators $a_{1}^{+}$and $a_{2}^{+}$. We again assume that the real potentials $V_{1,2}=V_{1,2}(x)$ are stationary but the intermediate real potential $V$ may depend on time as well and to which we may always add some arbitrary time-dependent function $\Delta=\Delta(t)$. In other words, we consider the following chain of Darboux-type transformations between the Schrödinger operators:

$$
S\left[V_{1}\right] \stackrel{a_{1}^{+}}{\longrightarrow} S[V] \quad \longrightarrow S[V+\Delta] \stackrel{a_{2}^{+}}{\longrightarrow} S\left[V_{2}\right] .
$$

As we have shown in Section 2 a first-order intertwining for $S\left[V_{1}\right]$ and $S\left[V_{2}\right]$ implies the existence of second-order symmetry operators, that is,

$$
\left[S\left[V_{1}\right], a_{1}^{+} a_{1}^{-}\right]=\left[S\left[V_{2}\right], a_{2}^{-} a_{2}^{+}\right]=0 .
$$

Let us first consider that case where $a_{1}^{+} a_{1}^{-}$is a trivial symmetry operator, i.e. $a_{1}^{+} a_{1}^{-}=$ $H_{1}+$ const. Then the intertwining operators $a_{1}^{ \pm}$being of the form (7) with superpotential (11) can be simplified to

$$
\rho(t) \equiv 1, \quad g(x, t) \equiv g(t), \quad h(x, t) \equiv h(x) .
$$

Thus the operators $a_{1}^{ \pm}$do not depend on time, $a_{1}^{ \pm}= \pm \partial_{x}+h^{\prime}(x)$. The same argumentation applies to the operators $a_{2}^{ \pm}$and $H_{2}$. Evidently, in this case the Hamiltonians $H_{1,2}$ belong to the class considered in [11], where it has been shown that reducible as well as irreducible second-order intertwining operators exist.

In the case of a non-trivial symmetry operator $a_{1}^{+} a_{1}^{-}$the potential $V_{1}$ is of the form (see [20] and references therein)

$$
V_{1}(x)=k_{0}+k_{1} x+k_{2} x^{2}+\frac{k_{3}}{\left(x+k_{4}\right)^{2}} .
$$

It is evident that this class of potentials does not exhaust all solutions of intertwining relations (37) with ansätze (38) or (53) for $q^{ \pm}$.

The conclusion drawn from this discussion is, that the intertwining relations investigated in this section have both reducible and irreducible solutions of second order in $\partial_{x}$.

\section{Second-order intertwining for non-stationary po- tentials}

In order to consider the intertwining of Schrödinger operators with a non-stationary potential, it is useful to simplify the general form (36) of the intertwining operator $q^{+}$a little further. Indeed, by inspecting the general intertwining relation (8) with $q^{+}$as given in (36) one finds the condition

$$
\operatorname{Im} F(x, t)=\frac{1}{4} \dot{g}(t) x+g_{1}(t)
$$


with an arbitrary real function $g_{1}$. After multiplying both sides of (8) with

$$
g^{-1 / 4}(t) \exp \left[i\left(\frac{\dot{g}(t) x^{2}}{8 g(t)}+\frac{g_{1}(t) x}{g(t)}-\int_{0}^{t} d t^{\prime} \frac{g_{1}^{2}\left(t^{\prime}\right)}{g^{2}\left(t^{\prime}\right)}\right)\right]
$$

and some algebra we obtain a new intertwining relation,

$$
\left(i \partial_{t}+2 i\left(\frac{\dot{g} x}{4 g(t)}+\frac{g_{1}}{g}\right) \partial_{x}-\tilde{H}_{1}\right) \tilde{q}^{+}=\tilde{q}^{+}\left(i \partial_{t}+2 i\left(\frac{\dot{g} x}{4 g}+\frac{g_{1}}{g}\right) \partial_{x}-\tilde{H}_{2}\right)
$$

which, of course, is equivalent to (8). Here the "new supercharge" and "new Hamiltonians" are given by

$$
\begin{aligned}
\tilde{q}^{+} & =g \partial_{x}^{2}-2(\operatorname{Re} F) \partial_{x}+B+\frac{i}{4} \dot{g}-\frac{1}{g}\left(\frac{\dot{g} x}{4}+g_{1}\right)^{2}-\frac{2 i F}{g}\left(\frac{\dot{g} x}{4}+g_{1}\right) \\
& \equiv g \partial_{x}^{2}-2(\operatorname{Re} F) \partial_{x}+\tilde{B}
\end{aligned}
$$

and

$$
\tilde{H}_{1,2}=H_{1,2}+\frac{1}{8}\left(\frac{\ddot{g}}{g}-\frac{\dot{g}^{2}}{2 g^{2}}\right) x^{2}+\frac{1}{2}\left(\frac{g_{1} \dot{g}}{g^{2}}-\frac{2 \dot{g}_{1}}{g}\right) x
$$

respectively.

Now, in a second step we make a non-local transformation [17] of the independent variables $(x, t) \mapsto(y, \tau)$ with

$$
\tau=\int_{0}^{t} d t^{\prime} \frac{1}{g\left(t^{\prime}\right)}, \quad y=g^{-1 / 2}(t) x-2 \int_{0}^{t} d t^{\prime} g_{1}\left(t^{\prime}\right) g^{-3 / 2}\left(t^{\prime}\right)
$$

which brings the intertwining relation (64) into the form

$$
\begin{aligned}
&\left(i \partial_{\tau}+\partial_{y}^{2}-g \tilde{V}_{1}\right)\left(\partial_{y}^{2}-2 g^{-\frac{1}{2}}(\operatorname{Re} F) \partial_{y}+\tilde{B}\right)= \\
&\left(\partial_{y}^{2}-2 g^{-\frac{1}{2}}(\operatorname{Re} F) \partial_{y}+\tilde{B}\right)\left(i \partial_{\tau}+\partial_{y}^{2}-g \tilde{V}_{2}\right)
\end{aligned}
$$

where

$$
\tilde{V}_{i}(y, \tau)=V_{i}(x, t)+\frac{1}{8}\left(\frac{\ddot{g}}{g}-\frac{\dot{g}^{2}}{2 g^{2}}\right) x^{2}+\frac{1}{2}\left(\frac{g_{1} \dot{g}}{g^{2}}-\frac{2 \dot{g}_{1}}{g}\right) x .
$$

This shows that, without loss of generality, we could have chosen from the very beginning the operator $q^{+}$to be of the form

$$
q^{+}(x, t)=\partial_{x}^{2}-2 f(x, t) \partial_{x}+b(x, t)+i c(x, t),
$$

with all coefficient functions being real-valued.

\subsection{Intertwining of a non-stationary Hamiltonian with a sta- tionary one}

In Section 2 we have only briefly mentioned that a first-order intertwining between Schödinger operators for a non-stationary potential $V_{1}$ and a stationary one $V_{2}$ is possible. Here, because of the absence of the $R$-separation of variables, we will reconsider this aspect for the case of a second-order intertwining operator of the general form (70) in more details. 
The intertwining relation (8) with ansatz (70) leads to the following conditions for the coefficient functions:

$$
\begin{aligned}
& \dot{f}=c^{\prime}, \quad \dot{b}+c^{\prime \prime}+4 c f^{\prime}=0, \quad f^{\prime \prime}-b^{\prime}-V_{2}^{\prime}+4 f f^{\prime}=0 \\
& \dot{c}+2 f V_{2}^{\prime}-b^{\prime \prime}-4 b f^{\prime}-V_{2}^{\prime \prime}=0, \quad V_{1}=V_{2}-4 f^{\prime} .
\end{aligned}
$$

It may easily be verified that a particular set of solutions of (71) can be expressed in terms of two arbitrary real-valued functions $f_{1}$ and $f_{0}$, which depend only on $x$ and $t$, respectively:

$$
\begin{aligned}
& f(x, t)=\frac{1}{2} \frac{f_{1}^{\prime}(x)}{f_{1}(x)+f_{0}(t)}, \quad c(x, t)=\frac{1}{2} \frac{\dot{f}_{0}(t)}{f_{1}(x)+f_{0}(t)}, \\
& V_{2}(x)=\frac{1}{f_{1}^{\prime 2}(x)}\left(\lambda_{0}^{2} \sigma \delta+\frac{1}{2}\left(f_{1}^{\prime}(x) f_{1}^{\prime \prime \prime}(x)-\frac{1}{2} f_{1}^{\prime \prime 2}(x)\right)-\frac{\lambda_{0}^{2}}{4} f_{1}^{2}(x)\right), \\
& V_{1}(x, t)=V_{2}(x)-4 f^{\prime}(x, t), \quad b(x, t)=f^{\prime}(x, t)+2 f^{2}(x, t)-V_{2}(x) .
\end{aligned}
$$

Recall that $b, c, f$ and $V_{1}$ are functions of $x$ and $t$ whereas $V_{2}$ is assumed to be stationary, i.e. independent of time $t$. This latter assumption actually leads to the explicit form $f_{0}(t)=\sigma \exp \left(\lambda_{0} t\right)+\delta \exp \left(-\lambda_{0} t\right)$ with arbitrary constants $\sigma, \delta$ and $\lambda_{0}$. For this case, the symmetry operators $R_{i}$ associated with the Schrödinger operators $S\left[V_{i}\right]$ read

$$
\begin{aligned}
& R_{1}=q^{+} q^{-}=H_{1}^{2}+\frac{1}{4} \lambda_{0}^{2}-2 \dot{c}-4 i c^{\prime} \partial_{x}-2 i c^{\prime \prime}, \\
& R_{2}=q^{-} q^{+}=H_{2}^{2}+\frac{1}{4} \lambda_{0}^{2},
\end{aligned}
$$

with $H_{i}=-\partial_{x}^{2}+V_{i}$. Note that $R_{1}$ explicitly depends on time, whereas $R_{2}$ is stationary, a fact, which helps to show that the transformed function $\psi_{1}=q^{+} \psi_{2}$ of a normalized solution of $S\left[V_{2}\right] \psi_{2}(x, t)=0$ is a normalizable solution of $S\left[V_{1}\right] \psi_{1}(x, t)=0$. Indeed, because of the linearity of the Schrödinger operator and the stationarity of $V_{2}$, we may set $\psi_{2}(x, t) \equiv \psi_{2}(x, t ; E)=e^{-i E t} \varphi_{E}(x)$ with $\varphi_{E}$ being an eigenstate of $H_{2}$ associated with eigenvalue $E$. Therefore, we have

$$
\left(\psi_{1}(x, t ; \tilde{E}), \psi_{1}(x, t ; E)\right) \equiv\left(q^{+} \psi_{2}(x, t ; \tilde{E}), q^{+} \psi_{2}(x, t ; E)\right)=\left(E^{2}+\lambda_{0}^{2} / 4\right) \delta_{\tilde{E} E} \cdot
$$

Finally, we mention that in the particular case of a constant potential $V_{2}$ (without loss of generality we may set $V_{2} \equiv 0$ ) the function $f_{1}$ has to be a solution of the linear fourth-order differential equation with constant coefficients (see eq. (72))

$$
f_{1}^{\prime \prime \prime \prime}-\lambda_{0}^{2} f_{1}=0
$$

whose general solution may easily be constructed by standard methods and will be an arbitrary linear combination of the fundamental solutions $\sin \left(\sqrt{\lambda_{0}} x\right), \cos \left(\sqrt{\lambda_{0}} x\right), \sinh \left(\sqrt{\lambda_{0}} x\right)$ and $\cosh \left(\sqrt{\lambda_{0}} x\right)$. Note that the partner potential $V_{1}(x, t)=-2\left[\partial_{x}^{2} \log \left(f_{1}(x)+f_{0}(t)\right)\right]$ has the same scattering properties as $V_{2} \equiv 0$. That is, it is a reflectionless potential. As particular example we may choose $f_{1}(x)=\cosh (x)$ and $f_{0}(t)=\cosh (t)$ which leads to $V_{1}(x, t)=-2 \cosh (x) \sinh (t) /(\cosh (x)+\cosh (t))^{2}$.

\subsection{Intertwining of a non-stationary Hamiltonian with a time- dependent harmonic-oscillator Hamiltonian}

In the remaining part of this section we will now consider the second-order intertwining of Schrödinger operators associated with two non-stationary Hamiltonians. In doing so 
we will limit ourselves to one of the simplest non-stationary Hamiltonians, namely, that of a harmonic oscillator with a time-dependent frequency and search for the class of non-stationary potentials $V_{1}$ which are intertwined with this one.

As a starting point we choose the potential to be of the form

$$
V_{2}(x, t)=-\frac{1}{8}\left(\frac{\ddot{g}(t)}{g(t)}-\frac{\dot{g}^{2}(t)}{2 g^{2}(t)}\right) x^{2},
$$

which corresponds to the trivial potential $\tilde{V}_{2} \equiv 0$ in $(69)$ as we also set $g_{1} \equiv 0$ in the following discussion. As a consequence we obtain from (68) the intertwining relation:

$$
\left(i \partial_{\tau}+\partial_{y}^{2}-\tilde{U}_{1}\right)\left(\partial_{y}^{2}-2 \tilde{f}(y, \tau) \partial_{y}+\tilde{B}(y, \tau)\right)=\left(\partial_{y}^{2}-2 \tilde{f}(y, \tau) \partial_{y}+\tilde{B}(y, \tau)\right)\left(i \partial_{\tau}+\partial_{y}^{2}\right)
$$

where

$$
\tilde{U}_{1}(y, \tau)=g(t) \tilde{V}_{1}(y, \tau), \quad \tilde{f}(y, \tau)=g^{-1 / 2}(t) \operatorname{Re} F(x, t)
$$

are given in terms of the new variables $y=x / \sqrt{g}$ and $\tau=\int_{0}^{t} d t^{\prime} g^{-1}\left(t^{\prime}\right)$. Taking into account the relations (72) we have

$$
\begin{array}{rlrl}
\tilde{U}_{1}(y, \tau) & =-4\left(\partial_{y} \tilde{f}\right)(y, \tau), & \tilde{B}(y, \tau) & =\left(\partial_{y} \tilde{f}\right)(y, \tau)+2 \tilde{f}^{2}(y, \tau)+i \tilde{c}(y, \tau) \\
\tilde{f}(y, \tau)=\frac{1}{2} \frac{\left(\partial_{y} \tilde{f}_{1}\right)(y)}{\tilde{f}_{1}(y)+\tilde{f}_{0}(\tau)}, & \tilde{c}(y, \tau)=\frac{1}{2} \frac{\left(\partial_{\tau} \tilde{f}_{0}\right)(\tau)}{\tilde{f}_{1}(y)+\tilde{f}_{0}(\tau)},
\end{array}
$$

where $\tilde{f}_{0}(\tau)=\tilde{\sigma} e^{\tilde{\lambda}_{0} \tau}+\tilde{\delta} e^{-\tilde{\lambda}_{0} \tau}$ and $\tilde{f}_{1}$ is an arbitrary solution of (75) with $\lambda_{0}$ and $x$ replaced by $\tilde{\lambda}_{0}$ and $y$, respectively. Returning back to original variables $(x, t)$ and setting $g(t) \equiv \rho^{2}(t)$, we find that the original potentials read

$$
\begin{aligned}
& V_{1}(x, t)=-\frac{\ddot{\rho}(t)}{4 \rho(t)} x^{2}-2\left[\partial_{x}^{2} \log \left(\tilde{f}_{1}\left(\rho^{-1}(t) x\right)+\tilde{f}_{0}\left(\int_{0}^{t} d t^{\prime} \rho^{-2}\left(t^{\prime}\right)\right)\right)\right], \\
& V_{2}(x, t)=-\frac{\ddot{\rho}(t)}{4 \rho(t)} x^{2}
\end{aligned}
$$

which may be compared with the result of Bluman and Shtelen [17] who derived new potentials being related to the free quantum system (in the $y$ - $\tau$ coordinates) via non-local transformations. Here we note that the special case $\rho(t)=\cos (\omega t)$ leads to the ordinary (i.e. time-independent) harmonic oscillator case and the corresponding transformation has been called Jackiw transformation.

The present result is also closely related to the special case $K^{\prime 2}-K^{\prime \prime}=$ const. discussed in Section 2.1 where we have shown its close connection with the R-separation of variables. Thus solutions for $V_{1}$ in (80) can be related to a free quantum system characterized by $(18)$.

\section{Concluding remarks}

In this paper we have considered the most general time-dependent first- and second-order intertwinings of non-stationary Schrödinger operators. Whereas, in the first-order case a

\footnotetext{
${ }^{5}$ See, for example, [22, 23] and [24] where more general transformations are given.
} 
complete discussion was possible, in the second-order case only particular examples have been discussed. These, in turn, are not as simple as those of the first-order case.

In the main text we have concentrated on the implications of the intertwining relations and did not take into account secondary aspects such as zero modes and domain questions, which we are going to discuss now. As already mentioned in the introduction, the intertwining may provide a new non-trivial solution $\psi_{1}=q^{+} \psi_{2}$ of a given problem only in the case when $\psi_{2}$ does not belong to the kernel of the intertwining operator $q^{+}$. Elements of the kernel of $q^{+}$lead to trivial solutions. On the other hand, $S\left[V_{1}\right] \psi_{1}=0$ may have non-trivial solutions which cannot be obtained from solutions of $S\left[V_{2}\right] \psi_{2}=0$. In this case, $\psi_{1}$ necessarily belongs to the kernel of the adjoint intertwining operator $q^{-}$, $q^{-} \psi_{1}=0$. Therefore, in order to find a complete set of solutions one has to consider also the kernel of the intertwining operator and that of the adjoint one. Again the first-order case allows a general discussion. The result of our discussion in that case (Section 2) has led us to the general form $q^{+}=\rho\left(\partial_{x}+h^{\prime}+i g^{\prime}\right)$, where $h$ and $g$ are given in (13). As $q^{+}$is a first-order differential equation the dimension of its kernel is at most one-dimensional:

$$
\psi_{2}(x, t) \propto \exp \{-h(x, t)-i g(x, t)\} .
$$

The restriction of solutions to the linear space of square-integrable functions then leads to the normalization condition

$$
1=\int d x\left|\psi_{2}(x, t)\right|^{2}=\int d y \exp \{-2 K(y)\} .
$$

Hence, the question of zero modes of $q^{+}$or its adjoint is equivalent to a discussion of broken versus unbroken SUSY of the corresponding stationary problem in the $y$-coordinate [3]. The situation in the case of second-order intertwining is a bit more complicated. However, it is clear that the corresponding kernels are at most two-dimensional. In the particular case studied in Section 4.1 the kernel of $q^{+}$is obviously empty for a real non-vanishing parameter $\lambda_{0}$ as in this case the symmetry operator $R_{2}=\left(q^{+}\right)^{\dagger} q^{+}$, cf. eq. (73), is strictly positive.

Another aspect which we did not discuss in the main text is concerned with domain questions. Indeed, one usually is interested only in square-integrable solutions of the Schrödinger equation. There is no guarantee that the intertwining operator maps a squareintegrable solution into another square-integrable one. This typically happens when the superpotential or, more generally, the coefficient functions of the intertwining operator become singular [3]. Hence, the rather general and abstract results presented in the main text should be understood in the sense that whenever there appear singular coefficient functions a careful analysis of such domain questions is mandatory. For example, the case of inverse-square potentials briefly mentioned in Section $3.1 \mathrm{~b}$ ) is only well-defined if the domain (Hilbert space) is defined by the space of square-integrable functions on the positive half-line with proper (e.g. Dirichlet) boundary conditions at $x=0$.

Finally, let us mention that it is also possible to extend our approach to complexvalued potentials $V_{1,2}$, which recently have attracted some attention [25]. The case of stationary complex potentials with first-order intertwining has already been studied in $[7$, 26]. However, because of the absence of the reality conditions, cf. eq. (12), the first-order intertwining of such potentials will not lead to the $R$-separation of variables. Furthermore, the corresponding Schrödinger operators are no longer self-adjoint and, therefore, no symmetry operators of the form $q^{+} q^{-}$and $q^{-} q^{+}$exist. This situation is similar to that 
of the Fokker-Planck and diffusion equation discussed in Section 2.2. For the higherorder intertwining the notion of irreducible transformations is lost in the case of complex potentials.

\section{Acknowledgements}

This work was partially (M.I. and D.N.) supported by RFBR. One of us (M.I.) is grateful to the DAAD for supporting a research visit to the University of Erlangen-Nürnberg and also likes to thank the INFN for supporting a subsequent visit to the University of Bologna. Kind hospitality of both institutions is also gratefully acknowledged.

\section{References}

[1] A. Andrianov, M. Ioffe and D. Nishnianidze, Phys. Lett. A 201 (1995) 103; Theor. and Math. Phys. 104 (1995) 1129; preprint solv-int/9810006.

[2] G. Darboux, Compt. Rend. Acad. Sci. (Paris) 94 (1882) 1456.

[3] G. Junker, Supersymmetric Methods in Quantum and Statistical Physics, (SpringerVerlag, Berlin, 1996).

[4] E. Schrödinger, Proc. Roy. Irish Acad. 46A (1940) 9; 46A (1941) 183; 47A (1941) 53.

[5] L. Infeld, Phys. Rev. 59 (1941) 737.

L. Infeld and T.E. Hull, Rev. Mod. Phys. 23 (1951) 21.

[6] G. Junker and P. Roy, quant-ph/9803024 to appear in Annals of Physics (1998).

[7] F. Cannata, G. Junker and J. Trost, Phys. Lett. A 246 (1998) 219.

[8] A.A. Andrianov, N.V. Borisov and M.V. Ioffe, Phys. Lett. A, 105 (1984) 19.

[9] A.A. Andrianov, N.V. Borisov, M.I. Eides and M.V. Ioffe, Phys. Lett. A 109 (1985) 143.

[10] A.A. Andrianov, M.V. Ioffe and V.P. Spiridonov, Phys. Lett. A 174 (1993) 273.

[11] A. Andrianov, F. Cannata, M. Ioffe and J.-P. Dedonder, Int. J. Mod. Phys. A 10 (1995) 2683.

[12] A.A. Andrianov, F. Cannata, M.V. Ioffe and D.N. Nishnianidze, J. Phys. A 30 (1997) 5037.

[13] V.G. Bagrov and B.F. Samsonov, Phys. Lett. A 210 (1996) 60.

[14] V.G. Bagrov and B.F. Samsonov, Phys. Part. Nucl. 28 (1997) 374.

[15] F. Finkel, A. González-López, N. Kamran and M.A. Rodríguez, preprint mathph/9809013. 
[16] N.G. van Kampen, Stochastic Processes in Physics and Chemistry, (North-Holland, Amsterdam, 1992).

[17] G. Bluman and V. Shtelen, J. Phys. A 29 (1996) 4473.

[18] W. Miller, Jr., Symmetry and Separation of Variables, (Addison-Wesley, London, 1977), chapter 2.1 .

[19] A. Galindo and P. Pascual, Quantum Mechanics I, (Springer-Verlag, Berlin, 1990), chapter 17 .

[20] W.I. Fushchych and A.G. Nikitin, J. Math. Phys. 38 (1997) 5944.

[21] E. Ince, Ordinary Differential Equations, (Dover Publ., New York, 1956).

[22] R. Jackiw, Ann. Phys. (NY) 129 (1980) 183.

[23] P.Y. Cai, A. Inomata and P. Wang, Phys. Lett. 91A (1982) 331.

[24] G. Junker and A. Inomata, Phys. Lett. 110A (1985) 195.

[25] See, for example, C.M. Bender and S. Boettcher, Phys. Rev. Lett. 80 (1998) 5243; J. Phys. A 31 (1998) L273.

[26] A. Andrianov, F. Cannata, J.-P. Dedonder and M.V. Ioffe, preprint SPbU-IP-97-24 (St. Petersburg Univ.) and quant-ph/9806019, to appear in Int. J. Mod. Phys. A. 